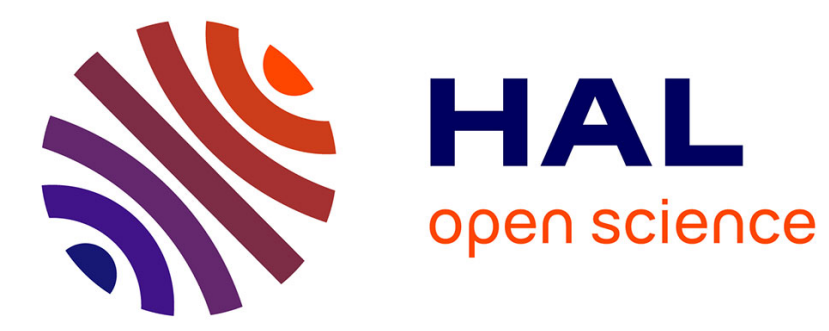

\title{
3D Deployment Problem in Wireless Sensor Networks resolved by genetic and ant colony algorithms
}

\author{
Sami Mnasri, Nasri Nejah, Malek Alrashidi, Thierry Val
}

\section{To cite this version:}

Sami Mnasri, Nasri Nejah, Malek Alrashidi, Thierry Val. 3D Deployment Problem in Wireless Sensor Networks resolved by genetic and ant colony algorithms. International Conference on Computer and Information Technology (ICCIT 2020), Feb 2020, Abuk (virtual Conference), Saudi Arabia. hal02969927

\section{HAL Id: hal-02969927 \\ https://hal.science/hal-02969927}

Submitted on 22 Oct 2020

HAL is a multi-disciplinary open access archive for the deposit and dissemination of scientific research documents, whether they are published or not. The documents may come from teaching and research institutions in France or abroad, or from public or private research centers.
L'archive ouverte pluridisciplinaire HAL, est destinée au dépôt et à la diffusion de documents scientifiques de niveau recherche, publiés ou non, émanant des établissements d'enseignement et de recherche français ou étrangers, des laboratoires publics ou privés. 


\section{D Deployment Problem in Wireless Sensor Networks resolved by genetic and ant colony algorithms}

\author{
Sami MNASRI \\ University of Toulouse \\ IRIT, RMESS \\ Toulouse, France \\ University of Gafsa \\ UPIM unit, Faculty of \\ Science of Gafsa \\ Gafsa, Tunisia \\ Sami.Mnasri@irit.fr
}

\author{
Nejah NASRI \\ University of Sfax \\ ENIS, LETI \\ Sfax, Tunisia \\ University of Tabuk, \\ Community College, \\ Department of computer \\ sciences Tabuk, KSA \\ nejah.nasri@isecs.rnu.tn
}

\author{
Malek ALRASHIDI \\ University of Tabuk, \\ Community College, Department \\ of computer sciences \\ Tabuk, KSA \\ mqalrashidi@ut.edu.sa
}

\author{
Thierry val \\ University of Toulouse \\ IRIT, RMESS \\ Toulouse, France \\ val@irit.fr
}

\begin{abstract}
Wireless Sensor Networks (WSN) is an evolving field of research with various applications. Deploying sensors is a decisive operation that greatly influences the performance and the functioning of the network. Comparing the 2D deployment with the 3D one, the latter is more complex because of its ability to attend more constraints imposed by real problems. On this article, we discuss the 3D node positioning problem, while minimizing the global number of sensors, the consumption levels of energy, and maximizing the covered area and the hybrid localization rate based on the 3D DV-HOP protocol and the RSSI. We seek to solve our problem by choosing the ideal 3D locations to add nomad nodes to achieve the mentioned objectives. An approach based on variants of optimization algorithms based on the NSGA-II algorithm [1] and the ACO algorithm [2] is proposed. The simulation model considers the implementation of the IEEE 802.15.4 standard, and a reactive AODV based routing layer. The results of simulations with OMNeT++ indicate the sustainability of the tested algorithms (NSGA-II, ACO).
\end{abstract}

Keywords ACO, Coverage, Deployment, FER, Localization, NSGA-II, RSSI

\section{INTRODUCTION}

Generally, WSN (wireless sensor networks) may be applied in most concerns needing monitoring tasks such as smart homes. The effectiveness of a WSN is closely related to the phase of repartitioning of sensors. Indeed, the strategy of deploying nodes seeks to precise the number and positions of nodes, and the network topology.

A WSN is qualified as three-dimensional if there is a non-negligible variation in the height of sensors compared to the width and length of the area of deployment. But most of the proposed deployment algorithms have been applied only to two-dimensional networks requiring disseminating the sensors on a $2 \mathrm{D}$ surface. In practical terms, ad hoc and wireless sensors are generally disseminated in a three dimensional area, such as aerial, underwater and underground networks.
The type of deployment of the nodes in the case of WSN is related to the application. In fact, some applications allow choosing the positions of sensors. The used deployment is named "deterministic". While in some other applications, nodes are distributed in a random way from an airplane or drone. The used deployment is named "non-deterministic". In accessible environments; since it requires fewer sensors than the random deployment; the deterministic one is privileged. However, the random deployment is generally privileged in the large-scale case.

The location of the sensors is the main factor in relation with the coverage problem. In WSN, the location details are indispensable if unusual events happen in the network.

Different objectives and parameters are taking into consideration such as network coverage, location, and consumption levels of energy. The majority of deployment models consider the problem of ideal positions of sensors as NP-hard. Hence, the difficulty of finding best solutions for this problem in its large-scale case, using deterministic classical methods. As a remedy, we suggest using an approach relying on the NSGA-II and ACO variants to resolve the positioning problem after the initial nondeterministic deployment. The suggested approach is distinguished from other models because it incorporates coverage optimization, localization, power consumption and lifetime into a single formulation.

The next sections of this paper are as following: Section 2 suggests an overview of 3D WSNs deployment. Specially, we present the NSGAII and ACO deployment algorithms and different modeling steps for WSNs. Section 3 explores our proposed approach for 3D deployment. Finally, section 4 presents OMNET++ simulations and discuss its results.

\section{RELATED WORKS}

Many recent researches propose approaches of optimizations in order to figure out the issues of $3 \mathrm{D}$ positioning of sensors. The objective of the deployment 
strategy relies on the scheduling activity in WSN according to the surface coverage criterion, is to optimize energy consumption and maximize network lifetime.

Geometric approach based on geometric calculations, example the Delaunay triangulation method [3] which aims to maximize the coverage of the area of interest with the existence of obstacles. The diagram of Voronoi [4], it is used to solve problems such as the deployment of sensors. In a $2 \mathrm{D}$ space, its goal is to maximize the coverage and lifetime of the WSN, and the virtual force that is used to address the problem of deploying sensors [5] [6].

Analytical approach based on mathematical modeling such as Meta-heuristics algorithms [7] which aims to solve many optimizations problems: Artificial Bee Colony $(\mathrm{ABC})$ its objective is to determine an optimal sensor distribution in an interest region (RoI) [8], the Particle Swarm Algorithm (PSO) which is an evolutionary algorithm inspired by the living world that utilizes a set of candidate solutions to identify a near to optimal solution for the problem of sensor deployment [9].

The evolutionary MOEA/D algorithm is based on decomposition to address multi-objective problems. Its operation is based on the creation of $\mathrm{N}$ single objective problems taking into account the initial multi-objective problem [10]. With the MOEA/D, each problem is divided into N scalar "sub-problems" classified by objective preferences and resolved simultaneously by evolutionary operators and information from neighbors.

Genetic algorithms (GA), which are stochastic algorithms based on natural evolution and genetics, among the, there is:

$\checkmark$ "Non-dominated Tri-Genetic Algorithm II" (NSGA-II) which is the most used algorithm to solve the problem of multi-objective optimization

$\checkmark$ "Non-Dominated Tri-Genetic Algorithm III" (NSGAIII), which is an NSGA-II enhancement, it utilizes a benchmark-based method to find solution for many multi-objective problems [11].

And there is the heuristic approach, which is an approximate method to solve an optimization problem which aims to find a feasible solution in a reasonable time of resolution.

\section{PROPOSED APPROACH}

Our proposed approach relies on the NSGA-II algorithm and the Ant Colony one.

\section{A. "Non-Dominated Genetic Sort Algorithm II" (NSGA-} II)

NSGA-II is a genetic algorithm where it is considered in the literature as one of the most efficient algorithms for solving a multi-objective optimization problem using the Pareto approach [12]. The NSGA-II consists of the following steps:

Step 0: Initialization: It consists in generating the initial population having $\mathrm{N}$ size in a random way.

Step 1: Evaluation: Evaluation of individuals: this is the calculation phase of the fitness function.

Step2: selection: the choice of individuals who will reproduce.
Step 3: Recombination: after the selection step, a recombination operation can be executed between a pair of parents. Then, the production phase of the descendants is achieved.

Step 4: mutation: the modification of a chromosome to improve the characteristics of the individual.

Step 5: The determination of a stopping criterion: We consider several stopping conditions to end the execution of the algorithm in order to ensure its convergence. That is, a maximum number of iterations are reached (set at 350 in our work); either a predefined coverage (or location) rate $\mathrm{k}$ is reached. Added to this, we use a maximum execution time. If it is reached, the algorithm stops.

\section{B. “Ant Colony Optimization” (ACO) Algorithm:}

ACO algorithms are meta-heuristics that are inspired by the behavior of ants. In the wild, if an ant has to choose between two paths with unequal lengths leading to a food source, it tends to choose the shortest one. The model explaining this behavior is as follows:

Step 1: An individual ant searching for food walks in random ways

Step 2: If this ant finds a "source" of food, it leaves a trail of "pheromones" in its path when coming back to the nest.

Step 3: These "pheromones" attract near ants which will to follow the "pheromones" track.

Step 4: Back to the nest, these same ants will strengthen the trail.

Step 5: To reach the same food source, if two tracks are possible, the shorter one will be covered by most ants.

Step 6: The short path will be stronger and therefore more attractive

Step 7: Since pheromones are volatile, the larger path will eventually disappear.

\section{Mathematical Model}

A mathematical formulation is suggested to solve the problem of 3D deployment of the sensor nodes. The goal is to identify a repartition schema of sensors while enhancing the coverage of the area. Besides, we want to optimize the localization of the sensors with the most uniformly distribution of them.

\section{$\checkmark$ Assumptions:}

The following hypotheses are supposed:

- An "anchor" node may be a sensor with a specific position. A "nomad" node is attached to a moving target.

- For the k-coverage case, the localized target should be in the range of $\mathrm{k}$ anchors or more.

- Following the used 3D location scheme relying on RSSI and DV-Hop measures, we need four anchors to locate each target. This 3D location scheme is detailed in section IV.

\section{$\checkmark$ Notation:}

The after-mentioned proposed notation relies on a set of parameters and decision variables.

- $\mathrm{T}$ symbolizes the $\mathrm{t}_{\mathrm{k}}$ targets detecting the region. 
$-\mathrm{N}$ depicts the sensor's types. $\mathrm{N}$ is the union of $\mathrm{Na}$ and $\mathrm{Nb}$ where $\mathrm{Na}$ describes the stationary node's types and $\mathrm{Nb}$ characterizes the mobile node's types.

-S symbolizes the set of possible sites where to deploy sensors. $\mathrm{S}$ is the union of $\mathrm{Sa}$ and $\mathrm{Sb}$ where $\mathrm{Sa}$ describes the stationary node's sites and $\mathrm{Nb}$ characterizes the mobile node's possible sites. A site should not simultaneously belong to the two sets $\mathrm{Sb}$ and $\mathrm{Sa}$.

$\checkmark$ Decision variables:

$-X_{t s}$ is a binary variable which is equal to ' 1 ' when a node $\mathrm{n} \in \mathrm{N}$ deployed on $\mathrm{s} \in \mathrm{S}$ can receive a signal coming from a target $\mathrm{t} \in \mathrm{T}$ with the minimum sufficient power.

$-M_{S}$ illustrates the minimum necessary hops needed to reach any mobile node by a stationary sensor located in $\mathrm{s} \in \mathrm{S}$.

$\checkmark$ Parameters:

- $\mathrm{n}$ describes the RoI length.

- m describes the RoI width.

- nbNa symbolizes number of stationary sensors.

- nbNm symbolizes number of appended mobile sensors.

- nbT indicates the target's number.

$\checkmark$ Objective functions

To formulate the coverage and the location of targets, we use the objective functions F1 and F2:

Coverage: we suggest the following F1 function for the coverage:

$$
F 1=\operatorname{maximiser}\left(\sum_{n_{m} \in N_{m}} F\left(n_{m}\right)\right)
$$

Where each position in the indoor $3 \mathrm{D}$ space must be monitored by at least $\mathrm{n}$ nodes to ensure complete coverage

Localization: we suggest the following F2 function for the location:

$$
\begin{gathered}
F 2=\operatorname{maximize} \sum_{t \in T}\left(\sum_{s \in S} X_{t s}-n_{\min }\right), \text { knowing that } \\
(x)^{+}=\max (0, x) \text { (2) }
\end{gathered}
$$

Where a target $t$ should be controlled by a minimum of $\mathrm{n}$ sensors to ensure a better location.

Then, the fitness function is given by:

$$
\begin{gathered}
F=F 1+F 2=\operatorname{maximize} \sum_{t \in T}\left(\sum_{s \in S} x_{t s}-\right. \\
\left.n_{\text {min }}\right)^{+}+\sum_{n \in N} F\left(n_{m}\right) \text { (3) }
\end{gathered}
$$

\section{SiMULATIONS USING OMNET ++}

In our simulation scenario, we aim to resolve the $3 \mathrm{D}$ deployment case using two, three and four objectives: the number of sensors, the coverage, the localization, and the power consumption levels.

In this regard, we used the following network architecture: we positioned 11 stationary sensors and three nomad ones to add. The stationary sensors positions are identified by the OMNet++ distribution law. The nomad sensors ones are identified using the genetic and ant algorithms. Likewise, for the mobile sensors, one is utilized to send the initial message.
The running scenario of simulations begins with a first message sent by the mobile sensor to a destination "d" randomly chosen. When the path to the sensor " $\mathrm{d}$ " is determined by the AODV protocol, this latter sensor becomes the new source and another destination is chosen. This loop is repeated until a fixed execution time of the algorithm is achieved.

As far as connectivity is concerned, a connectivity matrix between sensors relying on real prototyping is utilized. Hence, the connectivity connections values of the real experimental tests are injected firstly in the simulation model. Afterwards, to guarantee a dynamic scenario, the links of connectivity are disturbed by randomly modifying the RSSI values between sensors.

To determine the positions of sensors, we utilize the RSSI as a metric because the used localization model relies on hybridization between the RSSI protocol and the DV-Hop protocol. As a result, with higher value of RSSI, the location becomes more precise. A neighbor can enter the table of neighborhood of a sensor if and only if the RSSI values from the detected sensor exceeds a predefined threshold. Initially, this threshold is fixed at 100 . Then, to introduce a dynamic context, a disturbance of this value is incorporated using a random function $(+-30)$.

Figure 1 gives, for several numbers of purposes determined by the genetic algorithm, the average values of RSSI [13] of all sensors which are connected to the mobile sensor.

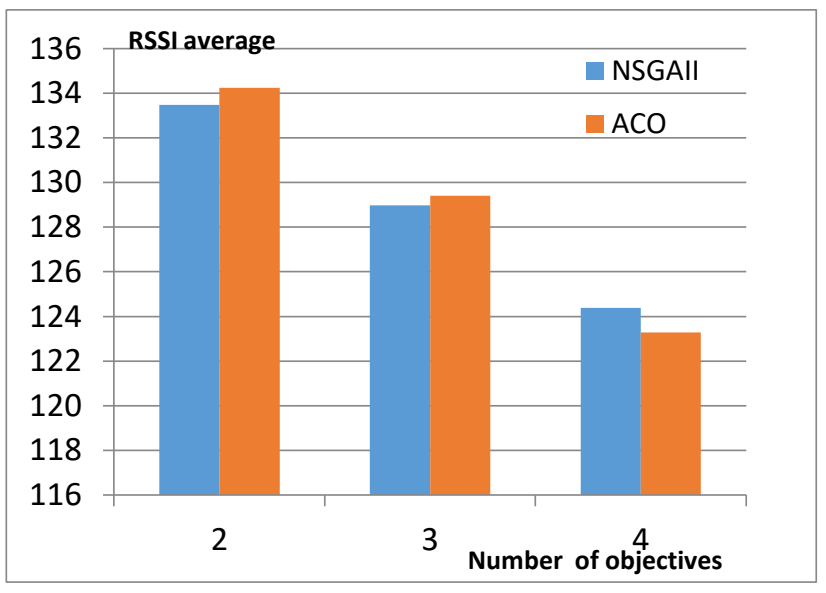

Fig. 1. Average RSSI rates in the simulations

To assess the coverage, the frame error level (FER) [14] is utilized as a measure of the quality of the different links between sensors. Hence, lower FER indicate a better coverage. Initially, FER rates are initialized to 0.01 . Then, to ensure dynamism on the execution scenario, a variation of the FER values is incorporated randomly in a field from 0.04 to 0.2 .

Figure 2 shows the FER average rates assessed for all sensors connected to the mobile node. 


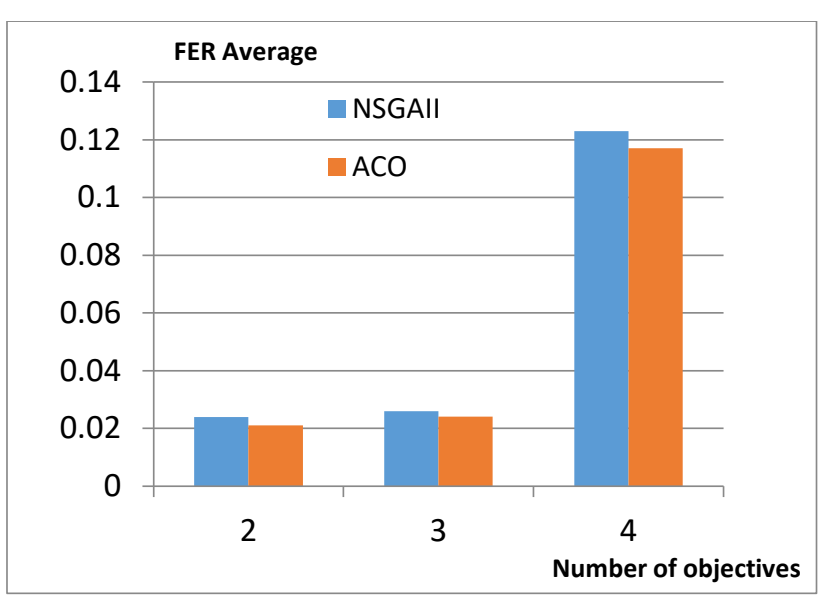

Fig. 2. Average FER rates in the simulations

To evaluate the connectivity level on the network, we assess the number of target neighbors. Figure 3 depicts the average number of neighbors of the mobile sensor per objective.

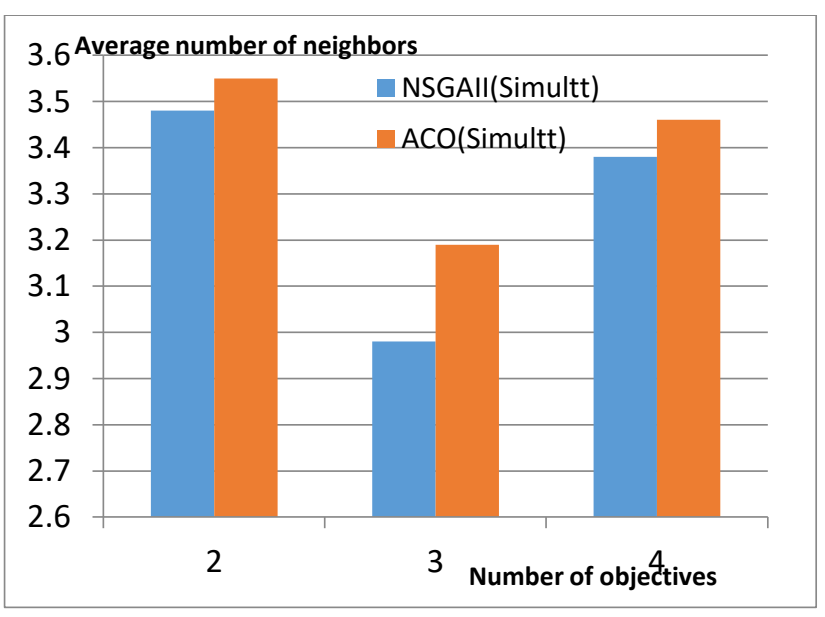

Fig. 3. Average number of neighbors in the simulations

The analysis of the simulations gives different interpretations:

The localization level, the coverage degree and the neighbor's number rises despite the decrease in RSSI rates for new nomad appended nodes compared to the RSSI rate of the initial nodes.

- Simulation figures indicate that if the objective's number do not exceed three, the NSGA II is more efficient than ACO. Yet, the ACO is better if the number of objectives is greater than three.

- We proved at the end of this work that the two chosen algorithms yielded effective results for our deployment problem. They allow resolving the deployment problem while maximizing location and coverage issues using the RSSI and FER metrics. Thus, they improved the connectivity links and the number of neighboring for mobile nodes.

\section{CONCLUSION}

The aim of this study is to give a 3D deployment schema for indoor wireless sensor networks taking into consideration several antagonist objectives. The considered objectives concern the simultaneous maximization of the coverage and the location according to the detection signal relying on a DV-Hop algorithm [15] enhanced by the RSSI information [16] ; and the minimization of the level of power consumption. We propose a strategy relying on a genetic algorithm (NSGAII) and ant colonies (ACO). We test our contributions with simulations using the OMNeT++ platform [17]. In the future, several research directions are worth of investigation. We can further improve the proposed method to consider the dynamic node redeployment [18] and take into account a variety of other goals, such as the global lifetime and the network utilization degree. In addition, we aim to propose a deployment hybrid approach combining the optimization theory [19] and other recent emergent paradigms and algorithms from the imagery treatment field [20][21].

\section{REFERENCES}

[1] Deb, K., Pratap, A., Agarwal, S., \& Meyarivan, T. A. M. T. (2002). A fast and elitist multiobjective genetic algorithm: NSGA-II. IEEE transactions on evolutionary computation, 6(2), 182-197.

[2] Dorigo, M. and G. Di Caro. "Ant colony optimization: a new meta-heuristic". in Proceedings of the 1999 congress on evolutionary computation-CEC99 (Cat. No. 99TH8406). 1999. IEEE.

[3] Nasri, N., A. Ben Fradj, and A. Kachouri, "Optimised crosslayer synchronisation schemes for wireless sensor networks". International Journal of Electronics, 2017. 104(7): p. 11781189.

[4] Okabe, A., et al., "Spatial tessellations: concepts and applications of Voronoi diagrams". Vol. 501. 2009: John Wiley \& Sons.

[5] Sangwan, A. and R.P. Singh, "Survey on coverage problems in wireless sensor networks". Wireless Personal Communications, 2015. 80(4): p. 1475-1500.

[6] Maheshwari, A.; Chand, N.: A survey on wireless sensor networks coverage problems. In: Krishna, C.; Dutta, M.; Kumar R. (eds.) Proceedings of 2nd International Conference on Communication, Computing and Networking. Lecture Notes in Networks and Systems, vol. 46. pp 153-164 (2019). Springer, Singapore. https://doi. org/10.1007/978-981-13-12175_16

[7] Mnasri S., Abbes F., Zidi K., Ghedira K. A multi-objective hybrid BCRC-NSGAII algorithm to resolve the VRPTW. 13th International Conference on Hybrid Intelligent Systems, Hammamet-Tunisia, 04- 06 December 2013

[8] Yu, X., et al., "A node deployment algorithm based on van der Waals force in wireless sensor networks". International Journal of Distributed Sensor Networks, 2013. 9(10): p. 505710.

[9] Sweidan, H.I. and T.C. Havens. "Coverage optimization in a terrain-aware wireless sensor network". in 2016 IEEE Congress on Evolutionary Computation (CEC). 2016. IEEE.

[10] Mnasri, S., Nasri N., and Thierry. V. "A hybrid ant-genetic algorithm to solve a real deployment problem: a case study with experimental validation". in International Conference on Ad-Hoc Networks and Wireless. 2017. Springer.

[11] Benatia, M.A., et al., "Multi-objective WSN deployment using genetic algorithms under cost, coverage, and connectivity constraints". Wireless Personal Communications, 2017. 94(4): p. $2739-2768$

[12] Mnasri S., Nasri N., Van Den Bossche A.,Val T. Improved many-objective optimization algorithms for the 3D indoor deployment problem. Arabian Journal for Science and Engineering. Doi: 10.1007/s13369-018-03712-7

[13] Li, K., et al., "An evolutionary many-objective optimization algorithm based on dominance and decomposition". IEEE Transactions on Evolutionary Computation, 2015. 19(5): p. 694-716. 
[14] Danping H., Portilla J., Riesgo T., "A 3D multi-objective optimization planning algorithm for wireless sensor networks,". 39th Annual Conference of the IEEE Industrial Electronics Society, IECON 2013, pp.5428-5433, November 10-13th , 2013. Doi: 10.1109/IECON.2013.6700019.

[15] Mnasri S., Nasri N., Val T. The deployment in the Wireless Sensor Networks: Methodologies, Recent Works and Applications. International Conference on Performance Evaluation and Modeling in Wired and Wireless Networks (PEMWN 2014), Tunisie - 04-07 November 2014

[16] Almutairi, Saad, S. Manimurugan, and Majed Aborokbah. "A new secure transmission scheme between senders and receivers using HVCHC without any loss." EURASIP Journal on Wireless Communications and Networking 2019.1 (2019): 88.

[17] Mnasri, S., Van Den Bossche A, Nasri N. and Val T. "The 3D Deployment Multiobjective Problem in Mobile WSN: Optimizing Coverage and Localization.". International Research Journal of Innovative Engineering. ISSN 2395-0560, Vol. 1 N. issue 5, May 2015.

[18] Xu H., Lai Z., Liang H., "A novel mathematical morphology based antenna deployment scheme for indoor wireless coverage,". IEEE 80th Vehicular Technology Conference (VTC Fall), p.1-5, 14-17 Sept. 2014. Doi: 10.1109/VTCFall.2014.6965828

[19] Mnasri S., Thaljaoui A., Nasri N., Val T. A genetic algorithmbased approach to optimize the coverage and the localization in the wireless audiosensors networks. IEEE International Symposium on Networks, Computers and Communications (ISNCC 2015), Hammamet, Tunisia, IEEExplore digital library, 13-15 May 2015.

[20] S.Manimurugan., K.Porkumaran., C.Narmatha .,"The New Block Pixel Sort Algorithm for TVC Encrypted Medical Image", Imaging Science Journal, Vol. 62 No.8, PP. 403-414, Sep- 2014

[21] Narmatha, C., P. Manimegalai, and S. Manimurugan. "A Lossless Compression Scheme for Grayscale Medical Images Using a P2-Bit Short Technique." Journal of Medical Imaging and Health Informatics 7.6 (2017): 1196-1204. 